\title{
Carotid Artery Intima-Media Thickness and Nonalcoholic Fatty Liver Disease Severity
}

Ana Cristina L Albricker ${ }^{1}$, Claudia A Couto ${ }^{2}$, Maria Carmo P Nunes ${ }^{2}$, Tâmara O Reis ${ }^{1}$, Maria Luiza RP Lima ${ }^{1}$, Paula Vieira T Vidigal ${ }^{3}$, Clara G Camelo ${ }^{4}$ and Teresa Cristina A Ferrari ${ }^{*}$

${ }^{1}$ Postgraduate Programme in Sciences Applied to Adult Health, Faculdade de Medicina, Universidade Federal de Minas Gerais, Belo Horizonte, Brazil ${ }^{2}$ Associate Professor, Departamento de Clínica Médica, Faculdade de Medicina, Universidade Federal de Minas Gerais, Belo Horizonte, Brazil

${ }^{3}$ Associate Professor, Departamento de Anatomia Patológica e Medicina Legal, Faculdade de Medicina, Universidade Federal de Minas Gerais, Belo Horizonte, Brazil ${ }^{4}$ Medical Course, Faculdade de Medicina, Universidade Federal de Minas Gerais, Belo Horizonte, Brazil

${ }^{5}$ Full Professor, Departamento de Clínica Médica, Faculdade de Medicina, Universidade Federal de Minas Gerais, Belo Horizonte, Brazil

\begin{abstract}
Background: Nonalcoholic fatty liver disease (NAFLD) has reached epidemic proportions in the last decade, and it is related to high cardiovascular mortality. This disorder encompasses a spectrum of increasingly severe clinicopathological conditions, i.e. nonalcoholic fatty liver (NAFL) and nonalcoholic steatohepatitis (NASH) with or without fibrosis/cirrhosis. Mortality associated with NAFLD is significantly higher in patients with the more advanced stages of this condition and is chiefly due to concomitant cardiovascular disease. The association between NAFLD severity and carotid artery intima-media thickness (CIMT) is controversial.
\end{abstract}

Objective: To investigate the association between CIMT and the clinical forms of NAFLD.

Methods: The study included 34 adult patients with NAFL, 20 with NASH, and 26 asymptomatic controls without the MS and/or NAFLD. The clinical and metabolic characteristics as well as CIMT values, measured using a fully automated ultrasound system, were compared between the NAFL and NASH groups. CIMT of the NAFLD patients were also compared to those of the control group.

Results: The proportion of patients with athermanous plaque was significantly higher in the NAFLD group in comparison to the controls. Internal carotid artery CIMT and the combined measurement in the right side (common carotid artery, carotid bifurcation and internal carotid) were higher in the NAFL group when compared to the NASH patients. No other significant differences were found between CIMT values of the NAFL and NASH groups. After adjustment for clinical, demographic, and laboratorial variables, age was the main determinant of CIMT.

Conclusions: No clinically significant association was observed between CIMT of the patients with NAFL and $\mathrm{NASH}$. Age was the main determinant of CIMT.

Keywords: Carotid artery intima-media thickness; Non-alcoholic fatty liver disease; Nonalcoholic fatty liver; Nonalcoholic steatohepatitis

\begin{abstract}
Abbreviations
AGA: American Gastroenterological Association; ALT: Alanine Aminotransferase; AP: Alkaline Phosphatase AST: Aspartate Aminotransferase; Bif: Carotid Bifurcation; BMI: Body Mass Index; CCA: Common Carotid Artery; CIMT: Carotid Artery Intima-Media Thickness; CVD: Cardiovascular Disease; DICOM: Digital Imaging And Communications In Medicine; GGT: Gamma-Glutamyl Transferase; HDL: High-Density Lipoprotein; HOMA: Homeostatic Model Assessment Index; ICA: Internal Carotid Artery; IQR: Interquartile Range; LDL: Low-Density Lipoprotein; MESA: Multi-Ethinic Study Of Atherosclerosis; MS: Metabolic Syndrome; NAFL: Nonalcoholic Fatty Liver; NAFLD: Nonalcoholic Fatty Liver Disease; SD: Standard Deviation; US: Ultrasound; US-CRP, Ultrasensitive C-Reactive Protein
\end{abstract}

\section{Introduction}

Nonalcoholic fatty liver disease (NAFLD) encompasses a spectrum of increasingly severe clinicopathological conditions - nonalcoholic fatty liver (NAFL) and nonalcoholic steatohepatitis (NASH) with or without fibrosis/cirrhosis - and is characterized by the deposition of fat in the liver in an amount exceeding $5-10 \%$ of its weight. This disorder is frequently associated with the metabolic syndrome (MS), which has led many authors to suggest that NAFLD represents the hepatic component of this syndrome. NAFLD has reached epidemic proportions in the last decade and is one of the most common causes of chronic liver disease $[1,2]$.
Mortality associated with NAFLD, 10-15 years after its diagnosis, is about $10-12 \%$, and is significantly higher in patients with the more advanced stages of this condition. In this same period of time, the risk of progression to cirrhosis is 5-10\%, and 1-2\% to hepatocellular carcinoma [2]. Increasing number of studies have shown that the higher mortality rate among NAFLD patients in comparison to the general population is chiefly due to concomitant cardiovascular disease (CVD) than does the progression of the liver disorder [3-6]. The strong association between NAFLD and MS may explain the high cardiovascular mortality observed in NAFLD patients [7]. Furthermore, accumulating evidence suggests that NAFLD is by itself a risk factor for coronary artery disease independently of established risk factors [8-11].

Increased carotid artery intima-media thickness (CIMT) is

*Corresponding author: Teresa Cristina A Ferrari, Departamento de Clínica Médica, Faculdade de Medicina, Universidade Federal de Minas Gerais, Av. Professor Alfredo Balena 190, 30130-100, Belo Horizonte, MG, Brazil, Tel: +55 31 3409 9746; Fax: +55 313409 9664; E-mail: tferrari@medicina.ufmg.br

Received July 24, 2014; Accepted August 28, 2014; Published September 04 2014

Citation: Albricker ACL, Couto CA, Nunes MCP, Reis TO, Lima MLRP, et al. (2014) Carotid Artery Intima-Media Thickness and Nonalcoholic Fatty Liver Disease Severity. J Cardiovasc Dis Diagn 2: 175. doi:10.4172/2329-9517.1000175

Copyright: (@ 2014 Albricker ACL, et al. This is an open-access article distributed under the terms of the Creative Commons Attribution License, which permits unrestricted use, distribution, and reproduction in any medium, provided the original author and source are credited. 
considered a surrogate marker of early generalized atherosclerosis and subclinical CVD [12]. There is evidence that NAFLD is associated with increased CIMT [13-15]. Recent studies addressed to investigate CIMT measured by conventional ultrasound (US) techniques, as an indicator of NAFLD severity have demonstrated controversial results $[16,17]$. Clarification of this issue may be of clinical importance in planning preventive and therapeutic strategies as well may help in the differential diagnosis between NAFL and NASH. Thus, considering this unresolved issue, we investigated the association between the clinical NAFLD forms - NAFL and NASH - and CIMT, measured by a newly developed fully automated US, which has been suggested by some authors to be more accurate than the conventional US methods $[18,19]$.

\section{Methods}

The study included 54 consecutive NAFLD outpatients ( 44 women and 10 men, mean age $54 \pm 11.3$ years) who were recruited from the Liver Disease Clinic of the University Hospital, Universidade Federal de Minas Gerais, Belo Horizontal, Brazil, between August/2010 and October/2011. Twenty-six age- and gender-balanced asymptomatic subjects without MS and/or NAFLD (17 women and 9 men, mean age $54 \pm 14.5$ years) who were referred to CMIT measurement for cardiovascular risk assessment were selected as controls. The study was approved by the Research Ethics Committee of the Institution and all the participants signed the informed consent term.

NAFLD was diagnosed according to the criteria of the American Gastroenterological Association (AGA): hepatic steatosis confirmed by US and/or liver biopsy, exclusion of other causes liver of disease (namely alcohol intake $>30 \mathrm{~g} /$ day for men and $>20 \mathrm{~g} /$ day for women, markers of chronic B and $\mathrm{C}$ hepatitis virus infections, auto-immune hepatic disorders, Wilson disease, hemochromatosis, and alpha-1antitripsin deficiency), no history of prior gastric or jejunoileal bypass, no exposure to hepatotoxins, and no use of any drug known to cause hepatic steatosis during the last six months [20].

MS was defined by the presence of 3 or more of the following conditions: abdominal obesity (waist circumference $\geq 90 \mathrm{~cm}$ in men and $\geq 80 \mathrm{~cm}$ in women), hypertriglyceridemia ( $\geq 150 \mathrm{mg} / \mathrm{dl}$ ), and low high-density lipoprotein (HDL)-cholesterol level $(<40 \mathrm{mg} / \mathrm{dl}$ in men and $<50 \mathrm{mg} / \mathrm{dl}$ in females), hypertension (systolic blood pressure $\geq 130$ $\mathrm{mmHg}$ and diastolic $\geq 85 \mathrm{mmHg}$ ) and fasting hyperglycemia $(\geq 100$ $\mathrm{mg} / \mathrm{dl})[21]$.

To analyze cardiovascular risk, we used the Framingham score. The Framingham risk equation was computed as the probability of developing a coronary event within 10 years, by gender, using the following parameters: age, total cholesterol, HDL-cholesterol, smoking, systolic blood pressure, diastolic blood pressure and diabetes mellitus. The patients were classified into three groups for cardiovascular risk in 10 years as follows: $<10 \%$ low risk, $\geq 10 \%$ and $\leq 20 \%$ intermediate risk, and $>20 \%$ high risk [22].

Individuals who performed at least mild exercise, characterized as daily routine activities, were considered physically active [23]. Smoking was defined as current or previous use of tobacco with no set time frame.

\section{Clinical and laboratorial investigation}

All patients underwent clinical, anthropometric, laboratory and US evaluations at inclusion in the study. Anthropometric data included: weight $(\mathrm{kg})$, height $(\mathrm{m})$, waist circumference $(\mathrm{cm}$; measured in a standing position midway between the lower limit of the rib cage and the iliac crest), and body mass index ([BMI]; weight $/ \mathrm{height}^{2}\left[\mathrm{~kg} / \mathrm{m}^{2}\right]$ ) [13]. For purposes of analysis, non-obese was defined as BMI $<30 \mathrm{~kg} /$ $\mathrm{m}^{2}$ and obese, BMI $\geq 30 \mathrm{~kg} / \mathrm{m}^{2}$. Waist circumference $\geq 90 \mathrm{~cm}$ in men and $\geq 80 \mathrm{~cm}$ in women was defined as high.

Laboratory assessment included: liver biochemistry, lipid profile, serum uric acid, TSH, and fasting serum insulin and glucose. Insulin resistance in non-diabetic patients was calculated using the homeostatic model assessment index $([\mathrm{HOMA}]$; serum insulin $[\mu \mathrm{U} / \mathrm{ml}] \times$ fasting glucose $[\mathrm{mmol} / \mathrm{l}] / 22.5)$, and insulin resistance was defined by HOMA values $\geq 3$ [24]. For analysis purposes, the total of patients with insulin resistance was counted by adding to the number of individuals with HOMA $\geq 3$, those with diabetes (patients on regular oral hypoglycemic and/or insulin, and/or fasting glucose $\geq 126 \mathrm{mg} / \mathrm{dl}$ evaluated at 2 different occasions).

Liver biopsy was indicated according to the following recommendations: differential diagnosis with other liver diseases when needed; assessment of prognosis in the presence of moderate or severe hepatic steatosis on US in the presence of predictors of NAFLD severity (obesity and/or type 2 diabetes and/or MS) [24-26]; persistently elevated levels of aminotransferases in spite of compliance with medication, and/or aspartate aminotransferase (AST)/alanine aminotransferase (ALT) ratio $>1$ [2]. For the histological analysis, we used the classification of Kleiner et al., whereby a score $>5$ confirms the diagnosis of NASH [27].

The diagnosis of NAFLD by US was based on standard criteria [28]. US were performed in all subjects using the same equipment and by the same operator, who was unaware of the clinical and laboratory results. The diagnosis of NASH was defined by the histological criteria and/or by the persistence of elevated levels of AST and/or ALT for a period equal to or longer than 6 months. For the cases in which time lapse between the biopsy and measurement of IMT was more than 6 months, sustained high aminotransferase levels were required to diagnose NASH.

\section{CIMT measurements}

Measurement of CIMT was performed by a fully automated US system, which employs radiofrequency signal tracking, using a portable high resolution US device (Esaote MyLab 30 Gold $^{\mathrm{mw}}$ Cardiovascular, Italy) with a $10 \mathrm{MHz}$ frequency linear transducer (LA332). All exams were performed by the same operator who was unaware of the diagnosis.

CIMT measurement was carried out according to a protocol previously described [29]. CIMT was measured automatically in the deeper wall of three segments of the right and left extracranial carotid arteries: at least $1 \mathrm{~cm}$ proximal to the dilation of the carotid bulb, $1 \mathrm{~cm}$ proximal to the flow divider (referred to as the bifurcation), and $1 \mathrm{~cm}$ distal to the flow divider in the internal carotid. The basic 2-dimensional mode image was used for the measurement. The arteries were evaluated along the longitudinal axis, where the arterial segment is more perpendicular to the US beam. The US dual line pattern was identified allowing the definition of the intima-media and media-adventitia interfaces. The distance between the 2 acoustic interfaces was taken as the CIMT measure. From the images obtained in the anterior, posterior or sternocleidomastoid accesses, the most horizontal one (straight line) with the best defined dual line pattern was selected for performing the measurement. We used RFQIMT software (Esaote SPA., Italy), which automatically measures CIMT in real time with direct analysis of the RF 
signal as reference. This signal is obtained with a wall tracking system that processes the raw RF signals that are received along a single line of observation (M-line processing). The CIMT value was defined as the average of 6 measurements at the location with better image resolution and less variability among the values of the measures (Figure 1). Images were acquired and stored in Digital Imaging and Communications in Medicine (DICOM) for further analyses, if necessary [30].

The CIMT values were analyzed by comparison with those of the control group, and also with reference values defined in large population studies. For the patients aged $\geq 45$ years, the reference values were those of the Multi-Ethinic Study of Atherosclerosis (MESA), which refers to the Hispanic population [31]; and for the individuals with age $<45$ years, those of the study conduct on French employees of an insurance company (AXA, Paris, La Defense, France) [30-33].

CIMT above the 75th percentile of the reference values of the population studies were considered high risk for CVD [34]. To evaluate the cardiovascular risk of each patient based on the 75th percentile, we selected the highest mean CIMT value for the right and left common carotid artery (CCA). Atheromatous carotid plaque was defined as CIMT values $\geq 1.5 \mathrm{~mm}$ [35]. We also analyzed combined measurements of CIMT characterized as follows: combined measurement-1 - the mean value of CIMT of the right and left CCA; combined measurement-2 mean of the total sum total of CIMT of the CCA, carotid bifurcation and the internal carotid artery on the right; combined measurement-3 - such as combined measurement-2, on the left.

\section{Statistical analysis}

Sample size calculation was based on the following parameters: alpha error of 0.05 , power of the test of 0.8 , and effect size of $0.2 \pm$ $0.2 \mathrm{~mm}$, taking into account the difference between CIMT of the NAFL and NASH groups. The sample size calculated using the above parameters was 39 and 14 patients with NAFL and NASH, respectively.

Categorical data were presented as numbers and percentages, continuous data were expressed as mean \pm standard deviation (SD) or median and interquartile range, depending on the pattern of distribution of each variable. To compare proportions, the chi-square test or the

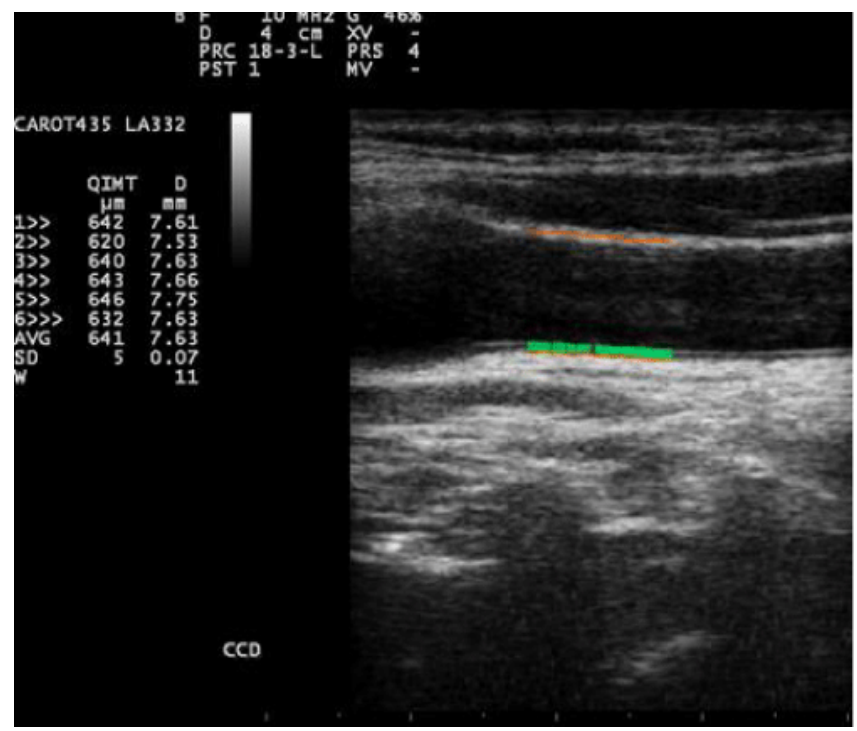

Figure 1: An example of intima-media thickness (common carotid artery) recording obtained by automated ultrasonograph.
Fisher's exact test were employed as appropriate. Continuous variables were compared between the groups using a t-test and ANOVA or the non-parametric Mann-Whitney and Kruskall Wallis tests as indicated.

Intra- and interobserver variabilities of CIMT of the right and left CCA were tested on 10 randomly selected cases, and the coefficient of variation was calculated.

In order to identify the predictor factors of CIMT, a multivariable linear regression analysis was performed, considering left CCA as a dependent variable. The potential predictors that were included into the multivariable model were NAFLD groups, age, gender, BMI, insulin resistance, and LDL cholesterol. The Statistical Package for the Social Sciences (SPSS) version 18 software (SPSS Inc. Chicago, IL) was used for statistical analysis. The values of $\mathrm{p} \leq 0.05$ were deemed statistically significant.

\section{Results}

Of the 54 NAFLD patients included in the study, 34 (63\%) were diagnosed with NAFL and $20(37 \%)$ with NASH. Of the 34 NAFL patients, the diagnosis of 8 (23.5\%) was confirmed by liver biopsy, while in the NASH group the diagnosis was established by biopsy in 14 cases $(70 \%)$. The mean age was $54.9 \pm 12.8$ years (range, $21-78$ years) in the NAFL group and $54.6 \pm 7.5$ years (range, $41-69$ years) in the NASH group ( $p=0.933)$. The results of the comparative analysis of the clinical and metabolic characteristics between the NAFL and NASH groups are described in Table 1. The proportions of patients with insulin resistance and with high serum levels of alkaline phosphatase and gamma-glutamyl transferase (GGT) were significantly higher in the NASH group. There was no other significant difference between these groups concerning the clinical and metabolic profile. The NAFLD group was similar to the controls regarding sex distribution $(\mathrm{p}=0.168)$ and median age $(\mathrm{p}=0.168)$.

All NAFLD patients underwent CIMT measurement as detailed below for the NAFL and NASH groups, respectively: CCA bilaterally, $34(100 \%)$ and $20(100 \%)$; right carotid bifurcation, $31(91.1 \%)$ and 14 (70\%); left carotid bifurcation, 22 (64.7\%) and 15 (75\%); right internal carotid artery, 22 (64.7\%) and 12 (60\%); left internal carotid artery, 21 (61.7\%) and 11 (55\%); combined measurement-1, $34(100 \%)$ and 20 (100\%); combined measurement-2, 25 (73.5\%) and 17 (85\%); combined measurement-3, $22(64.7 \%)$ and $16(80 \%)$. In the control group, the proportions of CMIT measurements in the different arterial segments were very similar to those of the NAFL group. Technical difficulties prevented the measurement of CIMT in all anatomic regions.

Table 2 presents the CIMT values and their comparative analysis between the NAFLD and control groups. The proportion of patients with atheromatous plaque was significantly higher in the NAFLD group. No other significant differences were observed between these groups.

The values of CIMT and their comparative analysis between the NAFL and NASH groups are described in Table 3. In both groups, CIMT values were below the 75 th percentile in approximately $60 \%$ of the patients. The CIMT of the right internal carotid artery and combined measurement- 2 were significantly higher in the NAFL group. Furthermore, there was a tendency of higher left CCA CIMT also in the NAFL group. No other significant differences were found between the NAFL and NASH groups. Atheromatous plaque was observed in around $26 \%$ of the NAFLD patients and there was no case with significant carotid stenosis. 
Citation: Albricker ACL, Couto CA, Nunes MCP, Reis TO, Lima MLRP, et al. (2014)Carotid Artery Intima-Media Thickness and Nonalcoholic Fatty Liver Disease Severity. J Cardiovasc Dis Diagn 2: 175. doi:10.4172/2329-9517.1000175

Page 4 of 6

\begin{tabular}{|c|c|c|c|}
\hline \multirow{2}{*}{ Variable } & \multicolumn{2}{|c|}{ Groups } & \multirow[b]{2}{*}{$P$ value } \\
\hline & NAFL $n / n(\%)$ & NASH n/n (\%) & \\
\hline Gender: Male & $5 / 34(14.7)$ & $5 / 20(25.0 \%)$ & $0.471^{*}$ \\
\hline Female & $29 / 34(85.3 \%)$ & $15 / 20(75.0 \%)$ & \\
\hline Hypertension & $21 / 29(72.4 \%)$ & $15 / 20(75.0 \%)$ & $0.840^{\dagger}$ \\
\hline Metabolic syndrome & $24 / 33(72.7 \%)$ & $15 / 19(78.9 \%)$ & $0.440^{*}$ \\
\hline increased waist circumference & $32 / 33(97.0 \%)$ & $19 / 19(100.0 \%)$ & $1.000^{*}$ \\
\hline Obesity (BMI $\geq 30$ ) & $19 / 33(57.6 \%)$ & $12 / 18(66.7 \%)$ & $0.525^{\dagger}$ \\
\hline Framingham risk score $>20 \%$ & $10 / 32(31.3 \%)$ & $7 / 20(35.0 \%)$ & $0.779^{\dagger}$ \\
\hline Physical activity & $16 / 31(51.6 \%)$ & $10 / 20(50.0 \%)$ & $0.910^{\dagger}$ \\
\hline Smoking & 9/31 (29.0\%) & 9/20 (45.0\%) & $0.244^{\dagger}$ \\
\hline Low HDL cholesterol & $19 / 34(55.9 \%)$ & $10 / 20(50.0 \%)$ & $0.675^{\dagger}$ \\
\hline LDL cholesterol $>100 \mathrm{mg} / \mathrm{dl}$ & $24 / 34(58.8 \%)$ & $14 / 20(70.0 \%)$ & $0.964^{\dagger}$ \\
\hline High triglycerides & $20 / 34(58.8 \%)$ & $9 / 20(45.0 \%)$ & $0.325^{\dagger}$ \\
\hline Resistance to insulin ${ }^{\ddagger}$ & $16 / 34(47.1 \%)$ & $18 / 20(90.0 \%)$ & $0.002^{*}$ \\
\hline AST/ALT >1 & $4 / 34(11.8 \%)$ & $5 / 20(25.0 \%)$ & $0.266^{*}$ \\
\hline High AP & $4 / 34(11.8 \%)$ & $8 / 20(40.0 \%)$ & $0.039^{*}$ \\
\hline High GGT & $17 / 34(50.0 \%)$ & $16 / 20(80.0 \%)$ & $0.029^{\dagger}$ \\
\hline High US-CRP & $19 / 27(70.4 \%)$ & $14 / 18(77.8 \%)$ & $0.735^{*}$ \\
\hline Statin use & $12 / 28(42.9 \%)$ & $4 / 20(20.0 \%)$ & $0.098^{\dagger}$ \\
\hline
\end{tabular}

Table 1: Comparative analysis of the clinical and metabolic characteristics and lifestyle between the nonalcoholic fatty liver and nonalcoholic steatohepatitis groups.

\begin{tabular}{|c|c|c|c|c|c|}
\hline \multirow{3}{*}{ Variable } & \multicolumn{4}{|c|}{ CIMT (mm) } & \multirow{3}{*}{$P$ value } \\
\hline & \multicolumn{2}{|c|}{ NAFLD group $(n=54)$} & \multicolumn{2}{|c|}{ Control group $(n=26)$} & \\
\hline & Mean \pm SD & Median/IQR & Mean \pm SD & Median/IQR & \\
\hline R CCA & $0.63 \pm 0.10$ & $0.63 / 0.12$ & $0.65 \pm 0.14$ & $0.63 / 0.20$ & $0.582^{*}$ \\
\hline L CCA & $0.69 \pm 0.16$ & $0.67 / 0.22$ & $0.62 \pm 0.15$ & $0.61 / 0.20$ & $0.610^{\dagger}$ \\
\hline R Bif & $0.62 \pm 0.12$ & $0.60 / 0.14$ & $0.60 \pm 0.17$ & $0.61 / 0.21$ & $0.731^{\dagger}$ \\
\hline LBif & $0.66 \pm 0.16$ & $0.64 / 0.25$ & $0.63 \pm 0.15$ & $0.60 / 0.25$ & $0.358^{*}$ \\
\hline R ICA & $0.53 \pm 0.10$ & $0.52 / 0.15$ & $0.53 \pm 0.16$ & $0.49 / 0.27$ & $0.853^{\dagger}$ \\
\hline L ICA & $0.50 \pm 0.11$ & $0.48 / 0.14$ & $0.44 \pm 0.13$ & $0.44 / 0.23$ & $0.078^{*}$ \\
\hline Combined-1‡ & $0.66 \pm 0.11$ & $0.66 / 0.16$ & $0.64 \pm 0.13$ & $0.64 / 0.20$ & $0.429^{*}$ \\
\hline Combined-2§ & $0.61 \pm 0.07$ & $0.61 / 0.09$ & $0.59 \pm 0.12$ & $0.59 / 0.18$ & $0.506^{*}$ \\
\hline \multirow[t]{2}{*}{ Combined-3" } & $0.64 \pm 0.15$ & $0.60 / 0.17$ & $0.56 \pm 0.09$ & $0.58 / 0.12$ & $0.720^{\dagger}$ \\
\hline & \multicolumn{2}{|c|}{ N/N (\%) } & \multicolumn{2}{|c|}{ N/N (\%) } & \\
\hline Percentile $\geq 75$ & \multicolumn{2}{|c|}{$22 / 54(41.7 \%)$} & \multicolumn{2}{|c|}{$6 / 26(23.1 \%)$} & $0.121^{\#}$ \\
\hline Ather plaque & \multicolumn{2}{|c|}{$14 / 54(25.9 \%)$} & \multicolumn{2}{|c|}{$0 / 26(0.0 \%)$} & $0.003^{\pi}$ \\
\hline
\end{tabular}

CIMT, carotid intima-media thickness; NAFLD, nonalcoholic fat liver disease; SD, standard deviation; IQR, interquartile range L, left; $R$, right; CCA, common carotid artery; Bif, carotid bifurcation; ICA, internal carotid artery; N/N, frequency found/ number of cases with available information; ather, atheromatous; "t-test; †MannWhitney; 趾C/LCC; \$ RCC/RBif/RCIA; "LCC/Lbif/LCIA; "chi-square test; "Fisher's exact test

Table 2: Carotid intima-media thickness of the carotid arteries and their comparative analysis between nonalcoholic fat liver disease and control group.

The comparative analysis between the NAFL and NASH groups concerning the cardiovascular risk according to the Framingham score is described in Table 4

Among clinical, demographic, and laboratorial variables, only age remained in the multivariable model as a predictor of CIMT (betacoefficient of $0.007 ; \mathrm{p}<0.001$ ) (Figure 2).

Intra- and interobserver variability for CIMT of the right CCA measurements were $4.1 \%$ and $8.7 \%$, and for CIMT of the left CCA were $4.5 \%$ and $8.9 \%$, respectively.

\section{Discussion}

In this study we evaluated CIMT in NAFLD patients and compared these values with those of asymptomatic controls, and also between the subjects with NAFL and NASH in order to investigate possible association between NAFLD severity and CIMT. We performed automatic measurement of CIMT using radiofrequency signal tracking, which has been considered an accurate method as the measurement is performed in real time, and during the procedure, it is necessary to correctly align the carotid walls, as well to respect the limits set for both number of measurements and variation among them [19,36,37]. To our best knowledge, this is the first study relating to NAFLD and CIMT measurements employing this technique.

\begin{tabular}{|c|c|c|c|c|c|}
\hline \multirow{3}{*}{ Variable } & \multicolumn{4}{|c|}{ CIMT (mm) } & \multirow{3}{*}{$\begin{array}{c}P \\
\text { value }\end{array}$} \\
\hline & \multicolumn{2}{|c|}{ NAFL group $(n=34)$} & \multicolumn{2}{|c|}{ NASH group $(n=20)$} & \\
\hline & Mean \pm SD & Median/IQR & Mean \pm SD & Median/IQR & \\
\hline R CCA & $0.65 \pm 0.12$ & $0.67 / 0.13$ & $0.62 \pm 0.08$ & $0.61 / 0.10$ & $0.263^{*}$ \\
\hline L CCA & $0.72 \pm 0.17$ & $0.71 / 0,19$ & $0.65 \pm 0.14$ & $0.61 / 0.17$ & $0.066^{\dagger}$ \\
\hline R Bif & $0.63 \pm 0.13$ & $0.61 / 0.15$ & $0.58 \pm 0.08$ & $0.58 / 0.10$ & $0.239^{\dagger}$ \\
\hline L Bif & $0.69 \pm 0.18$ & $0.70 / 0.28$ & $0.64 \pm 0.13$ & $0.62 / 0.13$ & $0.420^{*}$ \\
\hline R ICA & $0.55 \pm 0.10$ & $0.56 / 0.15$ & $0.49 \pm 0.09$ & $0.46 / 0.10$ & $0.028^{\dagger}$ \\
\hline LICA & $0.49 \pm 0.10$ & $0.47 / 0.14$ & $0.54 \pm 0.12$ & $0.52 / 0.16$ & $0.196^{*}$ \\
\hline Combined-1 $1^{\ddagger}$ & $0.68 \pm 0.12$ & $0.69 / 0.18$ & $0.63 \pm 0.10$ & $0.62 / 0.12$ & $0.118^{*}$ \\
\hline Combined- $2^{\S}$ & $0.63 \pm 0.07$ & $0.63 / 0.09$ & $0.58 \pm 0.07$ & $0.58 / 0.09$ & $0.023^{*}$ \\
\hline \multirow[t]{2}{*}{ Combined-3" } & $0.65 \pm 0.15$ & $0.61 / 0.21$ & $0.63 \pm 0.15$ & $0.60 / 0.10$ & $0.679^{\dagger}$ \\
\hline & \multicolumn{2}{|c|}{ N/N (\%) } & \multicolumn{2}{|c|}{$\mathrm{N} / \mathrm{N}(\%)$} & \\
\hline Percentile $\geq 75$ & \multicolumn{2}{|c|}{$14 / 34(41.2 \%)$} & \multicolumn{2}{|c|}{$8 / 20(40.0 \%)$} & $0.932^{\#}$ \\
\hline Ather plaque & \multicolumn{2}{|c|}{$8 / 34(23.5 \%)$} & \multicolumn{2}{|c|}{$6 / 20(30.0 \%)$} & $0.600^{\#}$ \\
\hline
\end{tabular}

CIMT, carotid intima-media thickness; NAFL, nonalcoholic fatty liver; NASH, nonalcoholic steatohepatitis; SD, standard deviation; IQR, interquartile range L, left; $\mathrm{R}$, right; CCA, common carotid artery; Bif, carotid bifurcation; ICA, internal carotid artery; N/N, frequency found/number of cases with available information ather, atheromatous; "t-test; ${ }^{\dagger}$ Mann-Whitney; $\neq$ RCC/LCC; \$RCC/RBif/RCIA; "LCC/ LBif/LCIA; "chi-square test

Table 3: Carotid intima-media thickness of the carotid arteries and their comparative analysis between nonalcoholic fatty liver and nonalcoholic steatohepatitis groups.

\begin{tabular}{|l|c|c|c|c|}
\hline \multirow{2}{*}{ Groups } & \multicolumn{3}{|c|}{ Framingham Score (risk) } & \multirow{2}{*}{ P value } \\
\cline { 2 - 5 } & Low & Intermediate & High & \\
\hline NAFL $(\mathrm{n}=32)$ & $16(50.0 \%)$ & $5(15.6 \%)$ & $11(34.4 \%)$ & $0.088^{*}$ \\
\hline NASH $(\mathrm{n}=20)$ & $6(30.0 \%)$ & $2(10.0 \%)$ & $12(60.0 \%)$ & \\
\hline
\end{tabular}

$\mathrm{NAFL}$, nonalcoholic fatty liver; $\mathrm{NASH}$, non-alcoholic steatohepatitis "chi-square test, lynear-By-linear association

Table 4: Framingham score and comparative analysis between nonalcoholic fatty liver and nonalcoholic steatohepatitis groups

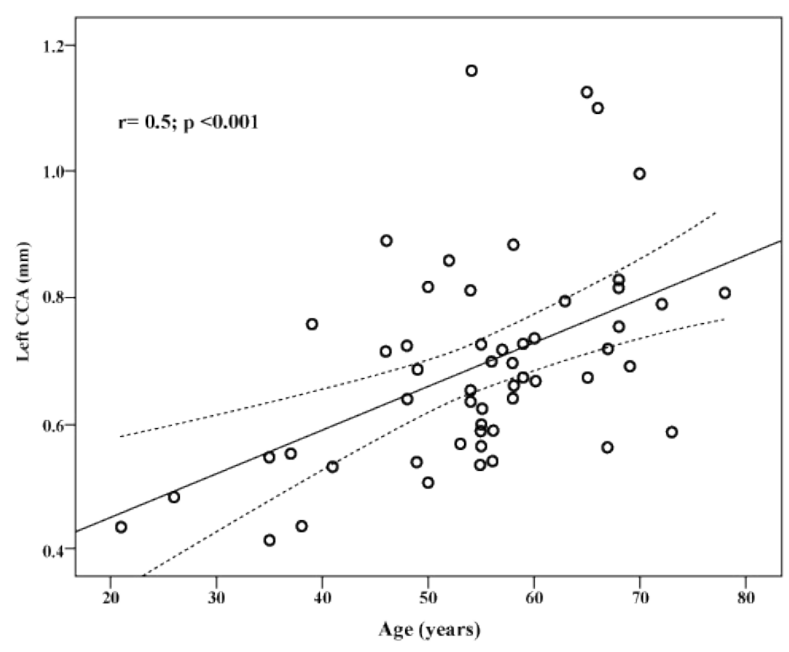

Figure 2: Scatter plot showing correlation between left common carotid artery intima-media thickness and age. 
The comparison of the carotid arteries examination between NAFLD patients and the control group showed only a higher proportion of atheromatous plaque in the NAFLD group. Concerning the comparative analysis between the NAFL and NASH groups, except for higher values of CIMT of the right internal carotid artery and combined measurement- 2 in the NAFL group, and a tendency of higher left CCA CIMT also in the NAFL group, no significant difference was found in any other arterial segments evaluated. Neither there was any difference between these groups when comparing the proportion of patients with CIMT higher than the values corresponding to the 75th percentile of the reference populations.

The relative lack of difference in CIMT between the NAFLD patients and the control group may be due to the fact the control group was not totally free of cardiovascular risk factors. Thus, some of the pro-atherogenic factors observed in the patients with NAFLD could also be present in the control group. Regarding the comparison between the NAFLD groups, contrary to the expectations, higher CIMT values were found in the NAFL patients; furthermore, the differences were observed only on one side. Thus, it is likely that these observed differences have been due to the relatively small sample size or just owing to chance. The use of statins could have been a confusing factor since the administration of these agents for longer than one year may stabilize or even reduce CIMT [38]. However, this possibility was ruled out as there was no difference between the proportions of patients with NAFL or NASH that did or did not take statin.

Clinical and metabolic changes often associated with the more severe NAFLD forms are obesity, age over 40-50 years, diabetes and arterial hypertension [39]. All these manifestations as well the MS were observed in most of our patients from both NAFLD groups. The similarity between the NAFL and NASH groups concerning the clinical and metabolic profile could have been the major cause for the lack of difference of the CIMT values between them. This similarity may be partly due to misclassification of some cases since the criteria to differentiate NAFL from NASH have limitations, even when based on liver biopsy, which is currently the more accurate method to diagnose the NAFLD clinical forms [40]. The more frequent occurrence of insulin resistance and higher serum levels of the canalicular enzymes (alkaline phosphatase and GGT) in the NASH group suggest that the majority of the cases were properly classified as NASH or NAFL. Insulin resistance plays an important role in the pathogenesis and progression of NAFLD; and, elevated liver enzymes is more frequent in the presence of more significant hepatic injury [41]. Including in the study only patients who underwent liver biopsy would certainly minimize the possibility of incorrect classification of the cases, but, on the other hand, could lead to the selection of more severe cases, since these patients fulfill more frequently the classical criteria for indicating liver biopsy.

In a study in which CIMT was measured by conventional US in 31 obese children and adolescents with NAFLD histologically diagnosed (19 with NAFL and 12 with NASH), and in 49 obese subjects without NAFLD, matched by sex, age and BMI, there was neither CIMT difference between the groups nor association between the histological pattern of NAFLD and CIMT [16]. On the other hand, in a study including 85 liver biopsy confirmed NAFLD patients and 160 individuals matched by gender, age and BMI without this diagnosis, Targher et al. found a strong association between NAFLD severity and increased CIMT measured by conventional US, and also with the presence of carotid atherosclerosis, independently of classical risk factors such as insulin resistance and MS. The 85 patients with NAFLD presented higher CIMT values in comparison with the 160 controls; and the 16 patients with NAFL presented lower CIMT values compared to the 69 subjects with NASH [17].

In predicting cardiovascular risk, a CIMT at or above the 75th percentile, as defined in large population studies, is considered to be high risk for CVD [29,30]. We expected higher CIMT values in the NASH group as these patients theoretically exhibit higher cardiovascular risk. However, most of our patients from both NAFLD groups presented CIMT values below the 75th percentile. The fact that this CIMT cutoff was defined in population studies in which the measurements were performed by conventional US systems, could explain, at least partially, our results. Another explanation related to the technique is the fact that we measured CIMT in the CCA at least $1 \mathrm{~cm}$ proximal to the origin of the bulb, where atheromatous plaque is less likely to be found $[42,43]$.

Even using the best image from the 3 angles, it was not possible to measure CIMT of the carotid bifurcation and internal carotid artery in a significant proportion of cases due to technical constraints, which did not occur in relation to the CCA. It is easier to obtain a straight line image of this arterial segment, which favors the positioning of the US beam perpendicularly to the artery wall. This aspect is reinforced by the lower range of the CIMT medians of the CCA $(9 \mathrm{~mm})$ compared to those of the carotid bifurcation $(13 \mathrm{~mm})$ and internal carotid $(10$ $\mathrm{mm}$ ). In the ARIC study, the number of valid measurements in the carotid bifurcation and internal carotid artery were also lower than those found in the CCA [29].

In our study, the proportion of patients classified as high risk according to the Framingham score was higher in the NASH group, although the difference was not statistically significant. Assessment of cardiovascular risk by the Framingham score and investigation of the occurrence of coronary artery disease over 10 years were the goals of a study that included 309 patients with NAFLD. Forty-one patients died, and coronary artery disease was the cause of death in 10 of them. The authors demonstrated that the Framingham risk score accurately predicted cardiovascular risk in 10 years in that population [44]. Considering that this score is a simple tool and easy to use in outpatients, we suggest adding the assessment of the cardiovascular risk by the Framingham score to the routine care of individuals with NAFLD.

In conclusion, no clinically significant association was found between CIMT and the NAFLD forms of presentation: NAFL and NASH. Except for the higher proportion of individuals with carotid atherosclerosis in the NAFLD group, CIMT of the control and NAFLD patients were also similar. The fact the control group was not totally free of cardiovascular risk factors, limitations concerning the differential diagnosis between NAFL and NASH, a relatively small sample size, and technical aspects of CIMT measurement may have exerted significant influences on the results; therefore, they should be interpreted with some caution. Studies with larger sample size in which liver biopsy is used to characterize the NAFLD forms are needed to clarify the existence or not of association between CIMT and NAFLD severity.

\section{References}

1. Targher G, Day CP, Bonora E (2010) Risk of cardiovascular disease in patients with nonalcoholic fatty liver disease. N Engl J Med 363: 1341-1350.

2. Schiff ER, Maddrey WC, Sorrell MF (2012) Schiff's diseases of the liver. (11 th Edn.), John Wiley \& Sons, Inc., Hoboken.

3. Ekstedt M, Franzén LE, Mathiesen UL, Thorelius L, Holmqvist M, et al. (2006) Long-term follow-up of patients with NAFLD and elevated liver enzymes. Hepatology 44: 865-873. 
Citation: Albricker ACL, Couto CA, Nunes MCP, Reis TO, Lima MLRP, et al. (2014)Carotid Artery Intima-Media Thickness and Nonalcoholic Fatty Liver Disease Severity. J Cardiovasc Dis Diagn 2: 175. doi:10.4172/2329-9517.1000175

Page 6 of 6

4. Marchesini G, Moscatiello S, Di Domizio S, Forlani G (2008) Obesity-associated liver disease. J Clin Endocrinol Metab 93: 74-80.

5. Targher G, Marra F, Marchesini G (2008) Increased risk of cardiovascula disease in non-alcoholic fatty liver disease: causal effect or epiphenomenon? Diabetologia 51: 1947-1953.

6. Rafiq N, Bai C, Fang Y, Srishord M, McCullough A, et al. (2009) Long-term follow-up of patients with nonalcoholic fatty liver. Clin Gastroenterol Hepato 7: 234-238.

7. Matteoni CA, Younossi ZM, Gramlich T, Boparai N, Liu YC, et al. (1999) Nonalcoholic fatty liver disease: a spectrum of clinical and pathological severity. Gastroenterology 116: 1413-1419.

8. Lin YC, Lo HM, Chen JD (2005) Sonographic fatty liver, overweight and ischemic heart disease. World J Gastroenterol 11: 4838-4842.

9. Hamaguchi M, Kojima T, Takeda N, Nagata C, Takeda J, et al. (2007) Nonalcoholic fatty liver disease is a novel predictor of cardiovascular disease. World J Gastroenterol 13: 1579-1584.

10. Mirbagheri SA, Rashidi A, Abdi S, Saedi D, Abouzari M (2007) Liver: an alarm for the heart? Liver Int 27: 891-894.

11. Targher G, Bertolini L, Padovani R, Rodella S, Zoppini G, et al. (2010) Prevalence of non-alcoholic fatty liver disease and its association with cardiovascular disease in patients with type 1 diabetes. J Hepatol 53: 713-718.

12. Stein JH, Fraizer MC, Aeschlimann SE, Nelson-Worel J, McBride PE, et al (2004) Vascular age: integrating carotid intima-media thickness measurements with global coronary risk assessment. Clin Cardiol 27: 388-392.

13. Alberti KG, Zimmet PZ (1998) Definition, diagnosis and classification of diabetes mellitus and its complications. Part 1: diagnosis and classification of diabetes mellitus provisional report of a WHO consultation. Diabet Med 15 $539-553$.

14. Expert Panel on Detection, Evaluation, and Treatment of High Blood Cholesterol in Adults (2001) Executive Summary of The Third Report of The National Cholesterol Education Program (NCEP) Expert Panel on Detection, Evaluation, And Treatment of High Blood Cholesterol In Adults (Adult Treatment Panel III). JAMA 285: 2486-2497.

15. Brea A, Mosquera D, Martín E, Arizti A, Cordero JL, et al. (2005) Nonalcoholic fatty liver disease is associated with carotid atherosclerosis: a case-control study. Arterioscler Thromb Vasc Biol 25: 1045-1050.

16. Manco M, Bedogni G, Monti L, Morino G, Natali G, et al. (2010) Intima-media thickness and liver histology in obese children and adolescents with nonalcoholic fatty liver disease. Atherosclerosis 209: 463-468.

17. Targher G, Bertolini L, Padovani R, Rodella S, Zoppini G, et al. (2006) Relations between carotid artery wall thickness and liver histology in subjects with nonalcoholic fatty liver disease. Diabetes Care 29: 1325-1330.

18. Caserta CA, Pendino GM, Amante A, Vacalebre C, Fiorillo MT, et al. (2010) Cardiovascular Risk Factors, Nonalcoholic Fatty Liver Disease, and Carotid Artery Intima-Media Thickness in an Adolescent Population in Southern Italy. Am J Epidemiol 171: 1195-1202.

19. Bianchini E, Bozec E, Gemignani V, Faita F, Giannarelli C, et al. (2010) Assessment of carotid stiffness and intima-media thickness from ultrasound data: comparison between two methods. J Ultrasound Med 29: 1169-1175.

20. Loria P, Adinolfi LE, Bellentani S, Bugianesi E, Grieco A, et al. (2010) The NAFLD Expert Committee of the Associazione Italiana per lo studio del Fegato (AISF). Practice guidelines for the diagnosis and management of nonalcoholic fatty liver disease: A decalogue from the Italian Association for the Study of the Liver (AISF) Expert Committee. Dig Liver Dis 42: 272-282.

21. Alberti KGMM, Eckel RH, Grundy SM, Zimmet PZ, Cleeman JI, et al. (2009) Harmonizing the Metabolic Syndrome A Joint Interim Statement of the International Diabetes Federation Task Force on Epidemiology and Prevention; National Heart, Lung, and Blood Institute; American Heart Association; World Heart Federation; International Atherosclerosis Society; and International Association for the Study of Obesity. Circulation 120: 1640-1645.

22. Wilson PW, D'Agostino RB, Levy D, Belanger AM, Silbershatz H, et al. (1998) Prediction of coronary heart disease using risk factor categories. Circulation 97: 1837-1847.

23. [No authors listed] (1998) American College of Sports Medicine Position Stand. The recommended quantity and quality of exercise for developing and maintaining cardiorespiratory and muscular fitness, and flexibility in healthy adults. Med Sci Sports Exerc 30: 975-991.

24. Haffner SM, Miettinen H, Stern MP (1997) The homeostasis model in the San
Antonio Heart Study. Diabetes Care 20: 1087-1092.

25. Rockey DC, Caldwell SH, Goodman ZD, Nelson RC, Smith AD; American Association for the Study of Liver Diseases (2009) Liver biopsy. Hepatology 49: 1017-1044.

26. Chalasani N, Younossi Z, Lavine JE, Diehl AM, Brunt EM, et al. (2012) The Diagnosis and Management of Non-Alcoholic Fatty Liver Disease: Practice Guideline by the American Association for the Study of Liver Diseases American College of Gastroenterology, and the American Gastroenterological Association. Hepatology 55: 2005-2023.

27. Kleiner DE, Brunt EM, Van Natta M, Behling C, Contos MJ, et al. (2005) Design and validation of a histological scoring system for nonalcoholic fatty liver disease. Hepatology 41: 1313-1321.

28. Sanyal AJ, American Gastroenterological Association (2002) AGA technical review on nonalcoholic fatty liver disease. Gastroenterology 123: 1705-1725

29. Howard G, Sharrett AR, Heiss G, Evans GW, Chambless LE, et al. (1993) Carotid artery intimal-medial thickness distribution in general populations as evaluated by B-mode ultrasound. ARIC Investigators. Stroke 24: 1297-1304.

30. Stein JH, Korcarz CE, Hurst RT, Lonn E, Kendall CB, et al. (2008) ASE consensus statement - Use of Carotid Ultrasound to Identify Subclinical Vascular Disease and Evaluate Cardiovascular Disease Risk: A Consensus Statement from the American Society of Echocardiography Carotid IntimaMedia Thickness Task Force Endorsed by the Society for Vascular Medicine. J Am Soc Echocardiogr 21: 93-111.

31. Bild DE, Bluemke DA, Burke GL, Detrano R, Diez Roux AV, et al. (2002) Multiethnic study of atherosclerosis: objectives and design. Am J Epidemiol 156 871-881.

32. Gariepy J, Salomon J, Denarié N, Laskri F, Mégnien JL, et al. (1998) Sex and Topographic Differences in Associations Between Large-Artery Wall Thickness and Coronary Risk Profile in a French Working Cohort: The AXA Study. Arterioscler Thromb Vasc Biol 18: 584-590.

33. Denarié N, Gariepy J, Chironi G, Massonneau M, Laskri F, et al. (2000) Distribution of ultrasonographically-assessed dimensions of common carotid arteries in healthy adults of both sexes. Atherosclerosis 148: 297-302.

34. Eleid MF, Lester SJ, Wiedenbeck TL, Patel SD, Appleton CP, et al. (2010) Carotid ultrasound identifies high risk subclinical atherosclerosis in adults with low framingham risk scores. J Am Soc Echocardiogr 23: 802-808.

35. Touboul PJ, Hennerici MG, Meairs S, Adams H, Amarenco P, et al. (2007) Mannheim carotid intima-media thickness consensus (2004-2006). An update on behalf of the Advisory Board of the 3rd and 4th Watching the Risk Symposium 13th and 15th European Stroke Conferences, Mannheim, Germany, 2004, and Brussels, Belgium, 2006. Cerebrovasc Dis 23: 75-80

36. Vanoli D, Lindqvist P, Wiklund U, Henein M, Näslund U (2013) Fully automated on-screen carotid intima-media thickness measurement: a screening tool for subclinical atherosclerosis. J Clin Ultrasound 41: 333-339.

37. Ciccone MM, Scicchitano P, Zito A, Agati L, Gesualdo M, et al (2011) Correlation between coronary artery disease severity, left ventricular mass index and carotid intima media thickness, assessed by radiofrequency. Cardiovasc Ultrasound 32: 1-8

38. Taylor AJ, Kent SM, Flaherty PJ, Coyle LC, Markwood TT, et al. (2002) Arteria Biology for the Investigation of the Treatment Effects of Reducing Cholesterol: A Randomized Trial Comparing the Effects of Atorvastatin and Pravastatin on Carotid Intima Medial Thickness. Circulation 106: 2055-2060.

39. Wieckowska A, Feldstein AE (2008) Diagnosis of nonalcoholic fatty live disease: invasive versus noninvasive. Semin Liver Dis 28: 386-395

40. Grandison GA, Angulo P (2012) Can NASH be diagnosed, graded, and staged noninvasively? Clin Liver Dis 16: 567-585.

41. Almeda-Valdés P, Cuevas-Ramos D, Aguilar-Salinas CA (2009) Metabolic syndrome and non-alcoholic fatty liver disease. Ann Hepatol 8: 18-24

42. Lau KH, Fung YK, Cheung YT, Tsang WK, Ying M (2012) Repeatability and reproducibility of ultrasonographic measurement of carotid intima thickness. $J$ Clin Ultrasound 40: 79-84.

43. Dogan S, Plantinga Y, Dijk JM, van der Graaf Y, Grobbee DE, et al. (2009) Manual B-mode versus automated radio-frequency carotid intima-media thickness measurements. J Am Soc Echocardiogr 22: 1137-1144.

44. Treeprasertsuk S, Leverage S, Adams LA, Lindor KD, St Sauver J, et al. (2012) The Framingham risk score and heart disease in nonalcoholic fatty liver disease. Liver Int 32: 945-950. 